\title{
Our mission statement
}

Published online: 9 November 2020

(c) Springer Nature Switzerland AG 2020

\begin{abstract}
This journal is committed to providing an editorially rigorous forum for the publication of academic research that aims to advance our collective understanding of society, and the relationships between individuals and their communities.
\end{abstract}

Keywords Anthropology $\cdot$ Communication \& media $\cdot$ Cultural studies $\cdot$ Gender studies · Human geography $\cdot$ Language \& linguistics $\cdot$ Law \& criminology $\cdot$ Political science $\cdot$ Race $\&$ ethnic studies $\cdot$ Sociology $\cdot$ Social policy $\cdot$ Urban studies

\section{The place of the social sciences}

The value of the social sciences in making tangible and immediate contributions to policy-making is indisputable. The knowledge ecosystems surrounding our most pressing areas of debate and societal concern-from political discourse, the climate emergency, and global health, to social and economic inequality and educational attainment - are profoundly enriched by the insights of social scientists and those in allied fields.

Increasingly, the complexities of the modern world necessitate the pooling of knowledge in the pursuit of new solutions to shared problems or interpretations of social phenomena. Here, social scientists are frequently seen as uniquely placed to act as the mediators and facilitators of discourse and interchanges between an array of different stakeholders, including policymakers, STEM academics, and other players.

Social scientists play a vital and fundamental scholarly function in sustaining a rigorous tradition of analysis and investigation. The social sciences form part of the bedrock of an open and evidence-based culture, in which academics and others have the freedom to shape and interrogate how we view, engage with and interpret the world around us. At its best, such a culture can create the conditions in which we can shape and imagine alternative futures.

This journal is committed to providing an inclusive forum for the publication of all such scholarship that seeks to enrich our collective understanding of society, and the relationships between individuals and their communities. 


\section{Editorial mission}

We are committed to publishing social science research of all types-whether theoretical, methodological, qualitative or quantitative-provided it meets our criteria for publication. As a broad-scope journal, we want to publish the scholarship that matters to each core field within our remit, whether it be niche in focus or of wider appeal and application, and irrespective to whether it represents a perceived incremental contribution or a more significant advance. We particularly welcome research that engages with urgent societal issues, such as those embodied in the UN's Sustainable Development Goals.

We recognise that research cannot be placed into neatly labelled 'disciplinary buckets', and that traditional boundaries are increasingly disappearing. We therefore encourage the submission of interdisciplinary scholarship that combines theory, methods or analytical frameworks from two or more disciplines. This could include, for instance, legal and social perspectives on science and technology, medical anthropology, environmental sociology and computational social sciences.

Article collections dedicated to topical and pressing areas of debate will be a regular feature in this journal. These collections will provide rich repositories for fresh thinking, and will cover research themes that may not find a natural home in more narrowly defined or niche journals. Our flexible publishing model means that these collections will have space to grow and evolve over extended periods of time.

\section{Editorial standards}

As an 'SN-prefixed' publication, this journal draws on the expertise and best practice from across all the imprints within the broad Springer Nature family. This includes a strong commitment to upholding high standards founded on robust editorial policies, underpinned by an efficient and fair peer review process.

We are committed to promoting transparency and reproducibility in academic research. Our data policy expects authors to share the data or materials that underpin their research, whether qualitative or qualitative in nature. Authors are required to provide a statement of data availability, outlining to readers how and where the data can be accessed. By requiring evidence of data sharing, we hope to encourage the open sharing, reuse and citation of social sciences data.

\section{Guiding philosophy}

We believe that genuine progress in understanding the world around us can only be achieved through a multi- and interdisciplinary endeavour. By championing and facilitating the publication of original research that enriches the body of knowledge across the social sciences, this journal hopes to make a valuable contribution to that effort. 